\title{
ON SPIN OF INTERACTING GRAVITON
}

V. UNT. INTERAKTEERUVA GRAVITONI SPINNIST

B. УНТ. О СПИНЕ ВЗАИМОДЕИСТВУЮЩЕГО ГРАВИТОНА

\section{(Presented by J. Einasto)}

1. It is generally believed that gravitation is the gauge field theory describing massless self-interacting bosons (gravitons) of spin 2. The aim of the present note is to show that no definite spin can be ascribed to (self)-interacting graviton, and according to the classification of field theories proposed by Y. Ogievetskij and I. Polubarinov [ $\left.{ }^{1}\right]$, gravitation belongs to class B. We shall analyze solutions of classical (non-quantized) field equations and demonstrate that in the approximation where radiation energy in the wave zone is taken into account, the Poincaré invariant projection operator should not be applied to decompose the tensor $\psi^{\mu v}$ of gravitational field into the irreducible representations.

Our main result can already be obtained by analyzing the wave zone solutions of the linearized Einstein-Maxwell equations (of the PauliFierz equations with sources in the form of electromagnetic radiation energy) ${ }^{*}$ :

$$
\square \psi^{\mu v}-\psi^{\mu \alpha}{ }_{, \alpha^{v}}-\psi^{v \alpha}{ }_{, \alpha^{\mu}}+\eta^{\mu v} \psi^{\alpha \beta}{ }_{, \alpha \beta}=2 x\left(T^{\mu v}+E^{\mu v}\right) .
$$

Here $E^{\mu v}$ is the energy momentum tensor of electromagnetic radiation, and $T^{\mu v}$ describes a bounded source. The result can be generalized to the case of self-interacting graviton in the full nonlinear Einstein theory where the role similar to that of electromagnetic radiation will be played by gravitational radiation.

2. In the Minkowski space, a general symmetric tensor is decomposable into a direct sum of subspaces, into the sum of one spin-2, one spin-1 and two spin-0 representations:

$$
\psi^{\mu v}=\left(P_{2}+P_{1}+P_{0}+P_{0^{\prime}}\right)_{\alpha \beta}^{\mu v} \psi^{\alpha \beta} .
$$

This decomposition and the appropriate projection operators have been exposed explicitly by Barnes [2]. In a coordinate representation the decomposition (2) is

$$
\psi^{\mu v}=\psi_{T T}^{\mu v}+\psi^{\mu, v}+\psi^{v, \mu}+\psi^{, \mu v}+\psi_{T}^{\mu v},
$$

where $\psi_{T T, v}^{\mu \nu} \equiv 0, \eta_{\mu v} \psi_{T T}^{\mu v} \equiv 0, \psi_{T, v}^{\mu \nu} \equiv 0, \psi^{\mu}, \mu \equiv 0$. Transverse traceless components $\psi_{T T}^{\mu \nu}$ describe the spin- $2, \psi_{T}^{\mu \nu}$ the spin- $0^{\prime}, \psi^{\mu}$ the spin-1 and $\psi$ the

* Here $\sqrt{-g} g^{\alpha \beta}-\eta^{\alpha \beta} \equiv \psi^{\alpha \beta}+O\left(\psi^{2}\right), g_{\alpha \beta}$ is the metric tensor, $g$ being its determinant; $\eta^{\alpha \beta}$ denotes the Minkowski metric, $f, \alpha \equiv \eta^{\alpha \beta} f_{, \beta}, f_{, \beta} \equiv \partial f / \partial x^{\beta}$. Greek indices take values $0,1,2,3$, Latin indices $-1,2,3$. Units will be used in which the velocity of light $c=1$ and the Newton gravitational constant $\gamma=1 ; x=8 \pi$. 
spin- 0 parts of the field. The physical meaning of $\psi_{T}^{\mu v}$. has been elucidated in a number of papers (see e. g. $\left[{ }^{3}\right]$ ), in this note we are interested in the existence of $\psi^{\mu}$ and $\psi$.

Let us first sketch proofs leading to the conventional interpretation of the spin of graviton. Let $E^{\mu v}=0$. By differentiating (2a) we get

$$
\begin{aligned}
& \square\left(\psi^{\mu}+\psi^{, \mu}\right)=\psi^{\mu}{ }_{v}, \\
& \square^{2} \psi=\psi^{\mu v}{ }_{, \mu v} .
\end{aligned}
$$

By applying formulae (2a), (3) and (4) for decomposing the conserved matter tensor in flat space-time, we have

$$
\begin{aligned}
& T^{\mu \nu}=T_{T T}^{\mu \nu}+T_{T}^{\mu \nu}+T^{\mu, v}+T^{v, \mu}+T_{, \mu \nu}, \\
& \square\left(T^{\mu}+T^{, \mu}\right)=T^{\mu \nu}, v=0, \\
& \square^{2} T=T^{\mu \nu}{ }_{, \mu \nu}=0,
\end{aligned}
$$

which together with appropriate boundary conditions yield $T=T^{\mu}=0$. Hence, in the subspaces of the fields of spins 1 and 0 , there exist no sources; $\psi^{\mu}$ and $\psi$ are «pure gauge» and we can take $\psi^{\mu}=\psi=0$. This means that graviton has no spin-1 or spin- 0 parts. This result is the consequence of conservation laws and is valid even in the case when there is no gauge invariance $\left[{ }^{4}\right]$.

The same result follows from the gauge invariance with respect to transformations $\mathrm{x}^{\mu^{\prime}}=\mathrm{x}^{\mu}+\xi^{\mu}$. The main argument goes as follows: $\psi^{\mu}$ and $\psi$ are $\xi^{\mu}$-dependent and by a proper choice of $\xi^{\mu}$, one can make them vanish.

Now, let

$$
E^{\mu v}=\varepsilon^{\mu \nu}(u, \vartheta, \varphi) / r^{2}+O\left(r^{-3}\right) .
$$

Usually an important difference between $T^{\mu v}$ and $E^{\mu v}$ is overlooked, and the Hilbert gauge $\psi^{\mu v}{ }_{v}=0$ is used to exclude the spin-0 and the spin-1 parts of the field and to reduce equations (1) to the following form

$$
\square \psi^{\mu v}=2 x\left(T^{\mu v}+E^{\mu v}\right) \text {. }
$$

These equations have an asymptotic solution $\left[{ }^{5}\right]$ (with an obscure physical content):

$$
\psi^{00}=1+(4 M+2 \ln r \Delta E) / r+\ldots,
$$

where $M$ is the mass of the source and $\Delta E$ denotes the finite amount of energy carried away by electromagnetic radiation. Let us characterize the function $f(\Omega)=\ln r \Delta E / r$ describing the gravitational field of electromagnetic radiation energy in the Hilbert gauge $(\Omega$ denotes the whole infinite wave zone region). The function $f(\Omega)$ has no Fourier transform, it does not belong to the class of functions for which the existence theorems for nonlinear hyperbolic equations can be proved, it does not satisfy appropriate boundary conditions. We can see that the operation $\square^{-1} E^{\mu \nu}$ is not defined within the reasonable class of functions. This implies that the projection operators that contain $\square^{-1}$ should not be applied to decompose either $E^{\mu v}$ or $\psi^{\mu v}$, otherwise we should obtain ill-defined field functions. In order to give a precise meaning to the word «ill-defined» we need some additional criteria that would enable us to define "good solutions» of equations (1). Here we shall make use of the Trautman boundary conditions, 
3. Trautman $\left.{ }^{6}\right]$ formulated the following boundary conditions to be imposed on gravitational field near the future null infinity: there exist coordinate systems and functions $b_{\mu \nu}=O\left(r^{-1}\right)$, such that

$$
\begin{gathered}
g_{\mu \nu}=\eta_{\mu \nu}+O\left(r^{-1}\right), \quad g_{\mu v, \lambda}=b_{\mu v} k_{\lambda}+O\left(r^{-2}\right), \\
\left(b_{\mu \lambda}-\frac{1}{2} \eta_{\mu \lambda} \eta^{\alpha \beta} b_{\alpha \beta}\right) k^{\lambda}=O\left(r^{-2}\right), \\
k^{\alpha} \equiv\left(1, x^{i} / r\right) .
\end{gathered}
$$

These conditions will be satisfied if the components of $\psi^{\mu \nu}$ in Cartesian coordinates have the following asymptotic form

$$
\psi^{\mu v}=a^{\mu v}(t-r, \vartheta, \varphi) / r+O\left(r^{-2}\right) .
$$

The only non-vanishing coefficients of $r^{-2}$ in (8) are

$$
\varepsilon^{00}=\varepsilon^{0 i} x^{i} / r=\varepsilon^{i k} x^{i} x^{k} / r^{2} .
$$

By inserting (8), (13) and (14) into (1), we can find the proper gauge and determine $\psi^{\mu v}{ }_{v}$ in terms of $\varepsilon^{00}$. The choice $\psi^{\mu v}{ }_{, v}$ is now clearly impossible: $\square \psi^{\mu v}=O\left(r^{-3}\right)$. It turns out that the Bondi gauge is the proper gauge. The solution of equations (1) in this gauge may be found in our paper $\left[{ }^{7}\right]$, here we reproduce the results only $(u \equiv t-r)$ :

$$
\begin{gathered}
a^{00}=a^{i 0} x^{i} / r=a^{i k} x^{i} x^{k} / r^{2}=2(M-E(u)), \\
E(u)=\int_{u_{1}}^{u} \int_{S} \varepsilon^{00} \sin \vartheta d \vartheta d \varphi d u,
\end{gathered}
$$

where $S$ denotes the surface of the unit sphere.

Similarily, we have found that the change of the asymptotic term in $\psi^{0 i}$ that describes the angular momentum of the source is determined by the flow of electromagnetic angular momentum.

Thus, $\psi^{\mu v}{ }_{v}$ has gauge-independent parts that describe the flow of energy and angular momentum. But this does not imply that gravitation has spin 0 and 1 parts. We have

$$
\psi^{0 v}, v=-2 r^{-2} E(u)+\ldots
$$

and equation (3)

$$
\square\left(\psi^{0}+\psi^{0}\right)=-2 r^{-2} E(u)
$$

has only an ill-defined solution. One can neither introduce the irreducible representations $\psi^{\mu}$ and $\psi$ of the Poincaré group, nor put $\psi^{\mu}=\psi=0$. The spin of the interacting graviton has no definite value.

\section{REFERENCES}

1. Ogievetskij, Y. I., Polubarinov, I. V. Ann. Phys., 25, № 3, 358-386 (1963)

2. Barnes, K. J. J. Math. Phys., 6, № 5, 788-794 (1965).

3. Fradkin, E. S., Vilkovisky, G. A. Phys. Lett., 73B, 209; 77B, 262 (1978).

4. Cavalleri, G., Spinelli, G. Riv. Nuovo Cimento, 3, № 8, 1-91 (1980); Appendix B.

5. Фок В. А. Теория тяготения, пространства и времени, § 87. М., ГИФ-МЛ, 1961.

6. Trautman, A. Lectures on General Relativity (mimeographed notes). London, King's College, 1958.

7. Kuusk, P., Unt, V. J. GRG, 7, № 5, 399-417 (1976).

Academy of Sciences of the Estonian SSR, Institute of Astrophysics and Atmospheric Physics
Received July 9,1987 